\title{
Effects of a slow harmonic displacement on an Atomic Force Microscope system under Lennard-Jones forces
}

\author{
Morad Khadraoui, Faouzi Lakrad ${ }^{\mathrm{a}}$, and Mohamed Belhaq \\ Laboratory of Renewable Energy and Dynamics of Systems, Faculty of Sciences Aïn Chock, \\ University Hassan II-Casablanca, Casablanca, Morocco
}

\begin{abstract}
We focus in this paper on the modeling and dynamical analysis of a tapping mode atomic force microscopy (AFM). The microbeam is subjected to a low frequency harmonic displacement of its base and to the Lennard-Jones (LJ) forces at its free end. Static and modal analysis are performed for various gaps between the tip of the microbeam and a sample. The Galerkin method is employed to reduce the equations of motion to a fast-slow dynamical system. We show that the dynamics of the AFM system is governed by the contact and the noncontact invariant slow manifolds. The tapping mode is triggered via two saddle-node bifurcations of these manifolds. Moreover, the contact time is computed and the effects of the base motion amplitude and the initial gap are discussed.
\end{abstract}

\section{Introduction}

The Atomic Force Microscope (AFM) is a scanning probe microscope that is used as a nano-scale tool for manipulation and characterization in nanosciences [1]. It can be used in a broad spectrum of applications such as imaging, nanolithography, electronics, chemical and biological analysis $[2,3]$. It is based mainly on a vibrating microcantilever with a nano-scale tip that interacts with a sample surface via intermolecular forces [4]. Indeed, understanding AFM vibrations is central to the correct interpretation of the AFM outputs. In the present paper, a Lennard-Jones (LJ) force [4] is used to model the tip-surface highly nonlinear interactions.

Several studies investigated the effects of resonant harmonic external and/or parametric forcings on an AFM subjected to the LJ forces [5-9] in the contact, noncontact and tapping modes. This latter was the dominant imaging mode for most scanning probe microscopes during the last decade [2]. It is based mainly on resonant excitations of the cantilever with a feedback loop keeping the cantilever vibration amplitude constant. This mode minimizes the shear forces, present in the contact mode, that can be destructive to the tip and samples. It overcomes some deficiencies of the noncontact mode by improving the resolution and enabling the measurement of mechanical properties [10]. However, operating the cantilever near its resonances can likely cause complex dynamics due to the nonlinearities. As a remedy nonresonant tapping mode techniques are used. Thus, AFM with low frequency excitation compared to the fundamental natural frequency of the cantilever is used, for instance, in the pulsed-force mode AFM [11] and the peak-force AFM [12]. Indeed, the low frequency excitation leads to lower the tapping force causing limited tip-sample contact areas and minimizing the loss of resolution.

The present work is focused on the dynamics of an AFM

a e-mail: f.lakrad@fsac.ac.ma system under LJ forces and a very slow harmonic base displacement. Consequently, the system can be viewed as a fast-slow system with two time scales dynamics: one ruled by natural frequencies of the system and the other by the low frequency of the base displacement. Solutions of the system follow the stable invariant slow manifolds in large regions of the phase space. For more informations on the fast-slow systems see for instance $[13,14]$. In fact, two stable invariant slow manifolds coexist, one corresponds to the contact mode and the other to the noncontact mode. These two stable slow manifolds undergo dynamic saddlenode bifurcations (through the collision with an unstable slow manifold) when the amplitude of the base displacement is varied. These dynamic bifurcations rule the contact time between the tip and the sample and they determine the operational mode of the AFM: contact, noncontact and tapping modes, respectively.

This work uses a continuous model of the AFM system and it can be viewed as a continuation of a previous paper by Lakrad [15] where a lumped mass model was used. The present paper is organized as follows: in section 2 we derive a continuous nonlinear model of the microcantilever under the LJ force and the base displacement using the Hamilton principle. Then, the static configurations and the corresponding natural frequencies and mode shapes are investigated in sections 3 . In section 4 , the Galerkin method is used to reduce the equations of motion to a fast-slow system. Then, the slow invariant manifolds are computed and the conditions of existence of the tapping mode and the contact time are determined.

\section{Mathematical modelling}

The classical beam theory based on the Euler-Bernoulli assumptions is used to develop a continuous model of an AFM probe, of length $L$, operating in air. As shown in Fig. $1, Z$ denotes the tip/sample separation distance in the reference configuration and $w(x, t)+y(t)$ indicates the to- 


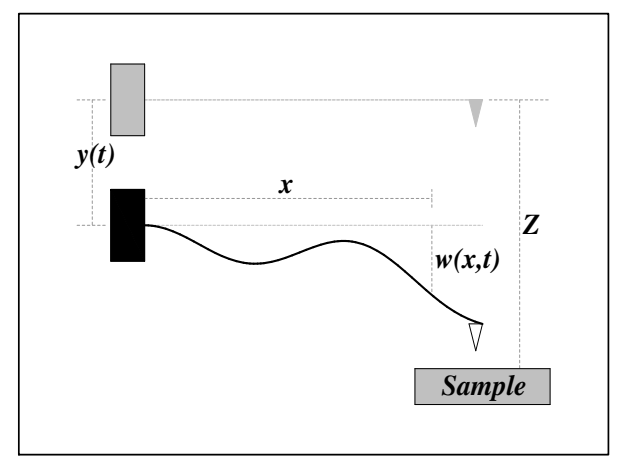

Fig. 1. A schematic of the AFM system

tal deflection of the microcantilever, where $w(x, t)$ is the deflection of the microcantilever relative to a non-inertial reference frame attached to the base. The base excitation from a dither piezo is assumed to be a vertical harmonic displacement; that is, $y(t)=Y^{*} \cos (\Omega t)$.

The cantilever-tip-sample interaction is modelled by a $\mathrm{LJ}$ force $F_{L J}$ between a sphere of radius $R$ and a flat surface $[4,7]$

$$
F_{L J}=\frac{A_{1} R}{180(Z-w(L, t)-y(t))^{8}}-\frac{A_{2} R}{6(Z-w(L, t)-y(t))^{2}}(1)
$$

where $A_{1}$ and $A_{2}$ are the Hamaker constants for the repulsive and attractive potentials, respectively. The attractive part of the $\mathrm{LJ}$ force corresponds to the van der Waals force [4]. In spite of its simplicity, this interaction model captures generic properties present in the near-field interactions [9]. In the present work, the $\mathrm{LJ}$ force $F_{L J}$ is assumed to be the unique source of nonlinearity of the system.

Using the Hamilton principle, the nondimensional form of the equation of motion and the associated boundary conditions are given by

$$
\begin{gathered}
\ddot{W}(X, \tau)+W^{\prime \prime \prime \prime}(X, \tau)+c \dot{W}(X, \tau)=-\ddot{Y}_{b}(\tau)-c \dot{Y}_{b}(\tau) \\
W(0, \tau)=0, \quad W^{\prime}(0, \tau)=0, \quad W^{\prime \prime}(1, \tau)=0
\end{gathered}
$$

and

$W^{\prime \prime \prime}(1, \tau)=\frac{-\Gamma_{1}}{\left(1-W(1, \tau)-Y_{b}(\tau)\right)^{8}}-\frac{\Gamma_{2}}{\left(1-W(1, \tau)-Y_{b}(\tau)\right)^{2}}(4)$

where the dots and the primes denote derivatives with respect to the nondimensional time $\tau=\omega t$ and the nondimensional space $X=x / L$, respectively. The displacements are normalized with respect to the initial gap $Z: W(x, t)=$ $w(x, t) / Z$ and $Y_{b}(t)=y(t) / Z=Y \cos (\Omega t)$. The other quantities are given by

$\omega^{2}=\frac{E I}{\rho A L^{4}} ; \quad c=\frac{c^{*}}{\rho A \omega} ; \quad \Gamma_{1}=\frac{-A_{1} R L^{3}}{180 Z^{9} E I} ; \quad \Gamma_{2}=\frac{A_{2} R L^{3}}{6 Z^{3} E I}$

in which $E, I, A$ and $\rho$ are, respectively, the cantilever modulus of elasticity, second moment of area, cross-section area, and material density. In addition, $c$ denotes the damping coefficient. The nondimensional instantaneous tip/sample separation is $\eta(1, \tau)=1-W(1, \tau)-Y_{b}(\tau)$.

Numerical applications are carried out for the case of the interaction of a soft monocrystalline silicon microcantilever

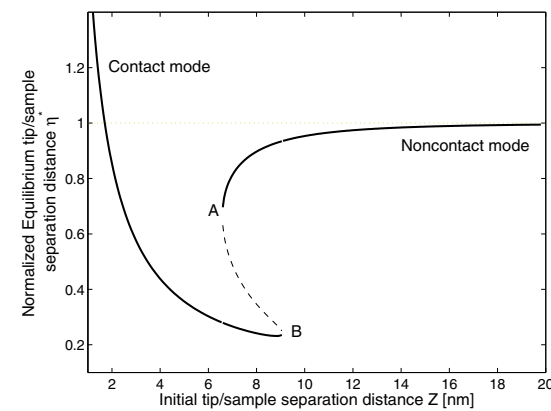

Fig. 2. Variation of the normalized tip/sample distance $\eta^{*}$ versus the initial separation $Z$

with the (111) reactive face of a flat silicon sample [9]. Indeed, such a soft cantilever can be used to minimize the tapping force and sample penetration, especially for soft samples. Moreover, in order to operate in the tapping mode it is recommended that the sample should have a low adhesion to the surface. All the key system parameters of the AFM system are listed in table 1.

Table 1. Properties and dimensions of the studied system [9]

\begin{tabular}{lll}
\hline Descriptions & Symbol & Values \\
\hline Length & $L$ & $449 \mu \mathrm{m}$ \\
Width & $\mathrm{b}$ & $46 \mu \mathrm{m}$ \\
Thickness & $\mathrm{h}$ & $1.7 \mu \mathrm{m}$ \\
Tip radius & $R$ & $150 \mathrm{~nm}$ \\
Material density & $\rho$ & $2330 \mathrm{~kg} . \mathrm{m}^{-3}$ \\
Elastic modulus & $E$ & $176 \mathrm{GPa}$ \\
Damping ratio & $\xi$ & 0.01 \\
Hamaker (rep.) & $A_{1}$ & $1.3596 \times 10^{-70} \mathrm{~J} \mathrm{~m}^{6}$ \\
Hamaker (att.) & $A_{2}$ & $1.865 \times 10^{-19} \mathrm{~J}$ \\
\hline
\end{tabular}

\section{Static and modal analyses}

In this section we solve the linearized undamped eigenvalue problem associated to Eqs. (2)-(4). For that, we first calculate static deflections $W^{*}(X)$ obtained from Eqs. (2)(4) by dropping the time derivatives and the base excitation. The static tip/sample separation distance $\eta^{*}$ is defined by $\eta^{*}=1-W^{*}(1)$, and is given by solving a ninth order algebraic equation.

In Fig. 2, we show the variation of $\eta^{*}$ versus the initial tip/sample separation $Z$. We can distinguish two regions: the first region corresponds to $Z<Z^{*}=1.7 \mathrm{~nm}$ where the system is repulsive since $\eta^{*}>1$. The second region corresponds to $Z>Z^{*}$ which is attractive (since $\eta^{*}<1$ ). In this second region, for $6.6 \mathrm{~nm}<Z<9.09 \mathrm{~nm}$, there is coexistence of three static solutions with two stable and one unstable.This region of bistability is located between the points $\mathrm{A}$ and $\mathrm{B}$ where saddle-node bifurcations occur. The lower and upper stable equilibria correspond, respectively, to the contact and noncontact configurations. Moreover, for high values of $Z$ the effect of the attractive van der Waals force becomes weaker till it asymptotically vanishes and consequently $\eta^{*} \rightarrow 1$. 
We compute the natural frequencies and mode shapes around a chosen static deflection $W^{*}(X)$ by setting

$$
W(X, \tau)=W^{*}(X)+V(X, \tau)
$$

Substituting Eq.(5) into Eqs. (2)-(4), dropping the damping and excitation terms and linearizing the outcome for small $V(X, \tau)$, then the free vibration of the cantilever is given by the following set of equations

$$
\ddot{V}(X, \tau)+V^{\prime \prime \prime \prime}(X, \tau)=0
$$

The boundary conditions are

$$
V(0, \tau)=0, \quad V^{\prime}(0, \tau)=0, \quad V^{\prime \prime}(1, \tau)=0
$$

and

$$
V^{\prime \prime \prime}(1, \tau)=-\left[\frac{8 \Gamma_{1}}{\left(1-W^{*}(1)\right)^{9}}+\frac{2 \Gamma_{2}}{\left(1-W^{*}(1)\right)^{3}}\right] V(1, \tau)
$$

Using separation of variables, the $\mathrm{n}^{\text {th }}$ mode shape is given by

$$
\begin{aligned}
\Phi_{n}(X) & =a_{n}\left[\cos \left(\lambda_{n} X\right)-\cosh \left(\lambda_{n} X\right)\right. \\
& \left.-\frac{\cos \left(\lambda_{n}\right)+\cosh \left(\lambda_{n}\right)}{\sin \left(\lambda_{n}\right)+\sinh \left(\lambda_{n}\right)}\left(\sin \left(\lambda_{n} X\right)-\sinh \left(\lambda_{n} X\right)\right)\right]
\end{aligned}
$$

where $a_{n}$ is computed by using the orthonormality condition of the modes, the nth natural frequencies coefficients $\lambda_{n}$ are zeros of the following algebraic equation

$$
\lambda_{n}^{3}=\beta \frac{\left[\cos \left(\lambda_{n}\right) \sinh \left(\lambda_{n}\right)-\sin \left(\lambda_{n}\right) \cosh \left(\lambda_{n}\right)\right]}{\left[1+\cos \left(\lambda_{n}\right) \cosh \left(\lambda_{n}\right)\right]} ;
$$

where $\quad \beta=-\left[\frac{8 \Gamma_{1}}{\left(1-W^{*}(1)\right)^{9}}+\frac{2 \Gamma_{2}}{\left(1-W^{*}(1)\right)^{3}}\right]$

The dimensional natural frequencies of the micro-cantilever are given by $\omega_{n}=\frac{\lambda_{n}^{2}}{L^{2}} \sqrt{\frac{E I}{\rho A}}$.

Fig. 3 displays the variations of the first two natural frequencies $\lambda_{1}$ and $\lambda_{2}$ with the initial tip/sample separation $Z$, for three different cases: the free-end microcantilever and the AFM microcantilever operating in the contact and non-contact states. For $Z<6.6 \mathrm{~nm}$, the AFM is in the contact mode, the frequencies are larger than in the case of the free end microcantilever because of the repulsive interactions. They are decreasing with increasing initial gap $Z$. In the bistable region $Z \in] 6.6 \mathrm{~nm}, 9.09 \mathrm{~nm}$ [, there is birth of a noncontact natural mode and an unstable mode (dashed red lines) through a saddle-node bifurcation. The noncontact mode is below the free-end mode and tends to it for increasing $Z$. The contact mode and the unstable mode disappear through a saddle-node bifurcation. For $Z \in] 9.09 \mathrm{~nm},+\infty[$ i.e., the noncontact mode, increasing $Z$ leads natural frequencies to tend toward the free-end natural frequencies. Moreover, the differences with the free end case frequencies are decreasing with increasing the order of the mode.

The two first mode shapes of the microcantilever under the LJ force are depicted in Fig. 4 for $Z=5 \mathrm{~nm}$ belonging to the monostable contact mode. These mode shapes are compared to the free-end mode shapes. It is observed that the first two mode shapes about the contact equilibrium are significantly different from those of the free-end case. On the other hand, the mode shapes about the noncontact static equilibrium are almost the same as those of the free-end microcantilever.

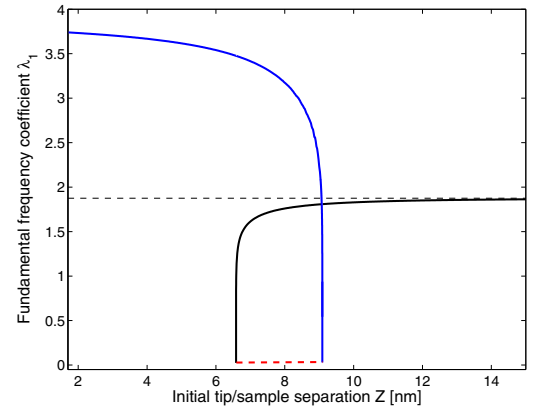

(a) First mode

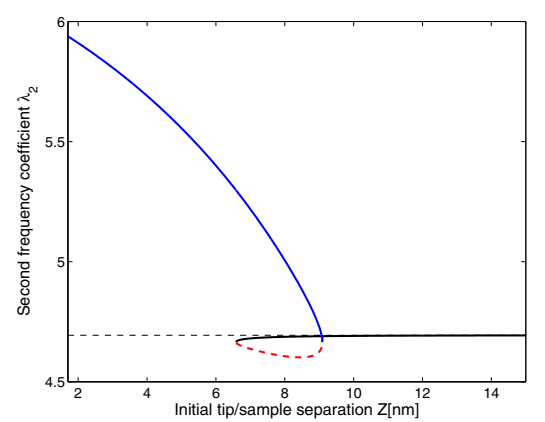

(b) Second mode

Fig. 3. Variation of the first two frequency coefficients $\lambda_{1}$ and $\lambda_{2}$, solution of Eq.(10), with the initial separation $Z$. The freeend microcantilever (dashed grey lines), the contact mode (blue), the noncontact mode (black) and the unstable mode (dashed red lines)

\section{Effects of the slow harmonic base motion}

In this section we will show that, due to the slow harmonic excitation of the base, the dynamics of the microcantilever can be reduced to the dynamics of the contact and the noncontact slow invariant manifolds. Moreover, based on the time spent on the attracting contact slow invariant manifold, the contact time during the tapping mode could be computed and controlled by the amplitude of the base displacement and the initial gap.

We use the Galerkin procedure to reduce the partial differential equation (2) and the associated boundary conditions into a set of nonlinear ordinary differential equations. In what follows only the first mode will be considered, thus

$$
W(X, \tau)=W^{*}(X)+q_{1}(\tau) \Phi_{1}(X)
$$

Then, the extended Hamilton principle and the orthonormality property of the mode shapes (9) are applied to obtain the following discretized equation

$$
\begin{aligned}
& q_{1}^{\prime \prime}+\xi q_{1}^{\prime}+\left[\lambda_{1}^{4}+\Phi_{1}^{2}(1)\left(\frac{8 \Gamma_{1}}{\left(1-W^{*}(1)\right)^{9}}+\frac{2 \Gamma_{2}}{\left(1-W^{*}(1)\right)^{3}}\right)\right] q_{1} \\
+ & \Phi_{1}(1)\left[\frac{\Gamma_{1}}{\left(1-W^{*}(1)\right)^{8}}+\frac{\Gamma_{2}}{\left(1-W^{*}(1)\right)^{2}}\right] \\
- & \frac{\Gamma_{1} \Phi_{1}(1)}{\left(1-W^{*}(1)-q_{1} \Phi_{1}(1)-Y_{b}(\tau)\right)^{8}} \\
- & \frac{\Gamma_{2} \Phi_{1}(1)}{\left(1-W^{*}(1)-q_{1} \Phi_{1}(1)-Y_{b}(\tau)\right)^{2}}=-\alpha_{1}\left(c Y_{b}^{\prime}(\tau)+Y_{b}^{\prime \prime}(\tau)\right)
\end{aligned}
$$




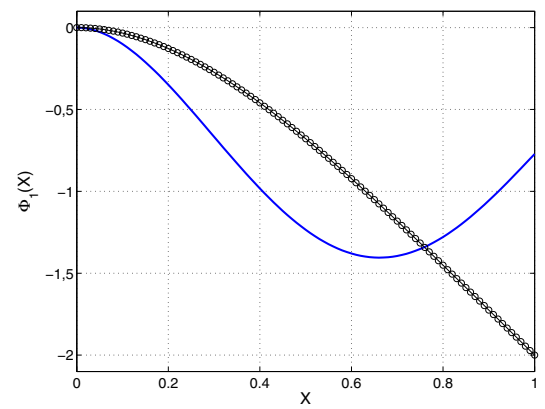

(a) Mode shape 1

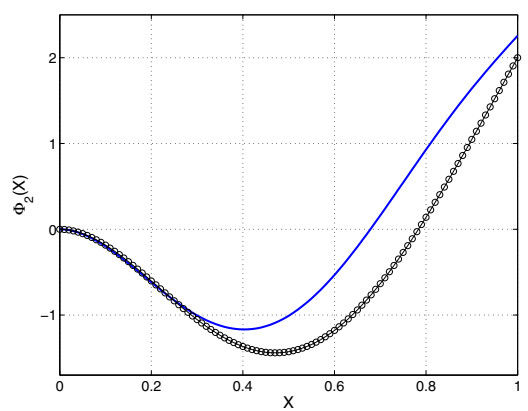

(b) Mode shape 2

Fig. 4. The first two modes shapes in the contact mode for $Z=5$ $\mathrm{nm}$. The blue line corresponds to the microcantilever with LJ force and the line with circles corresponds to the free end microcantilever

where the primes denote derivatives with respect to $\tau$ and the base motion $Y_{b}(\tau)=Y \cos (\epsilon \tau)$ with $\epsilon=\Omega / \omega<<1$. Furthermore,

$$
\xi=\frac{c^{*}}{\rho A} ; \quad \alpha_{1}=\int_{0}^{1} \Phi_{1}(X) d X .
$$

\subsection{Invariant slow manifolds}

Equation (12) can be transformed to a fast-slow system by introducing the slow time scale $T=\epsilon \tau$. Following Fenichel's results [13] there exist stable slow invariant manifolds $\mathcal{M}$ such that all orbits, starting in some region of the phase space, will reach them and the dynamics of the system is reduced to the dynamics in $\mathcal{M}$. A first order approximation of the invariant slow manifolds, corresponding to $\epsilon=0$ in the fast-slow system, is given by the following algebraic equation

$$
\begin{aligned}
& -\left[\lambda_{1}^{4}+\Phi_{1}^{2}(1)\left(\frac{8 \Gamma_{1}}{\left(1-W^{*}(1)\right)^{9}}+\frac{2 \Gamma_{2}}{\left(1-W^{*}(1)\right)^{3}}\right)\right] q_{1}(T) \\
- & {\left[\frac{\Gamma_{1} \Phi_{1}(1)}{\left(1-W^{*}(1)\right)^{8}}+\frac{\Gamma_{2} \Phi_{1}(1)}{\left(1-W^{*}(1)\right)^{2}}\right] } \\
+ & \frac{\Gamma_{1} \Phi_{1}(1)}{\left(1-W^{*}(1)-q_{1}(T) \Phi_{1}(1)-Y \cos (T)\right)^{8}} \\
+ & \frac{\Gamma_{2} \Phi_{1}(1)}{\left(1-W^{*}(1)-q_{1}(T) \Phi_{1}(1)-Y \cos (T)\right)^{2}}=0
\end{aligned}
$$

Hence, the approximation of $\mathcal{M}$ is given by the graph of a function of the generalized coordinate $q_{1}(T)$ in terms of

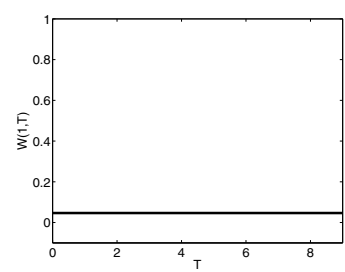

(a) $Y=0$

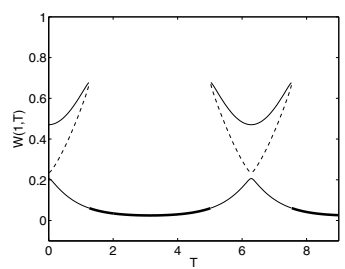

(c) $Y=0.345$

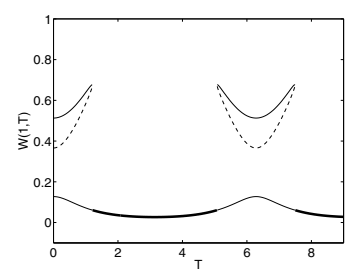

(b) $Y=0.3$

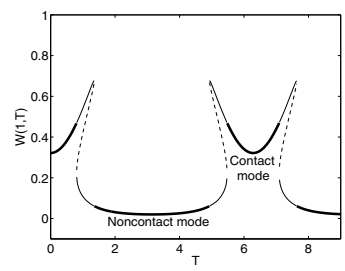

(d) $Y=0.5$
Fig. 5. Graphs of the invariant slow manifolds, given by Eq.(14), for $Z=10 \mathrm{~nm}$ and for various amplitude of the base $Y$.

$T$ and the parameters of the system. In Fig. 5 are shown the graphs of the slow manifolds versus the slow time $T$, for various values of the amplitude $Y$ and for the initial gap $Z=10 \mathrm{~nm}$. The stable manifolds are plotted with continuous lines and unstable ones with dashed lines. When only one stable manifold exists, it is plotted with a thick continuous line. The lower and the upper stable solutions correspond to the noncontact and contact slow manifolds, respectively. For $Y=0$ i.e., no base motion, the slow manifold corresponds to the noncontact static equilibrium which is independent of $T$. For $Y=0.3$, a reverse saddle-node bifurcation gives birth to two slow manifolds and the noncontact manifold is the visited solution. Then, for $Y=$ 0.3452 a second saddle-node bifurcation is taking place since the noncontact slow manifold collides with the unstable manifold. Subsequently, for $Y=0.5$ the contact and the noncontact slow manifolds are both visited during a period of the base displacement. Physically, this corresponds to the tapping mode and geometrically to a periodic burster. Figure 6 confirms that numerical solutions of Eq.(12) follow the slow invariant manifolds according to the scenario described in Fig 5.

\subsection{Contact time}

The contact time $t_{c}$ is the time that the tip is interacting repulsively with the sample [16]. It depends on the mechanical properties of the sample and the tapping amplitude and frequency. In our case, the tapping frequency is equal to the base excitation frequency and is very low than the fundamental frequency of the microcantilever. Indeed, the tapping mode in our case is governed by saddle-node bifurcations of slow manifolds, consequently the contact duration can be computed as the interval of time starting when the contact slow manifold is attracting and ending when it is repelling. In Fig. 7 are shown the normalized contact time versus the amplitude of the base motion $Y$ for various $Z$ that are initially in the noncontact mode i.e., $t_{c}=0$. The contact time is normalized by the period of the slow base displacement. Indeed, increasing $Z$ requires higher amplitude $Y$ to trigger the tapping mode. Moreover, a nontrivial 


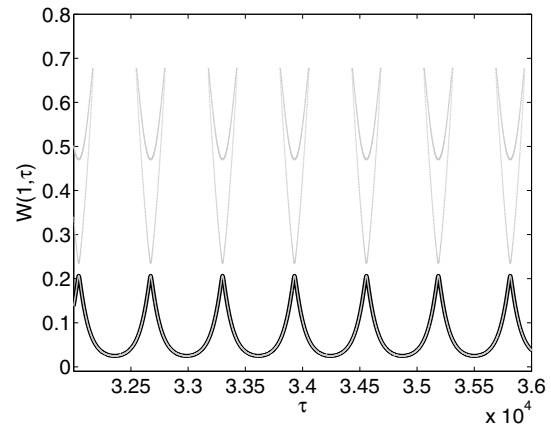

(a) $Y=0.345$

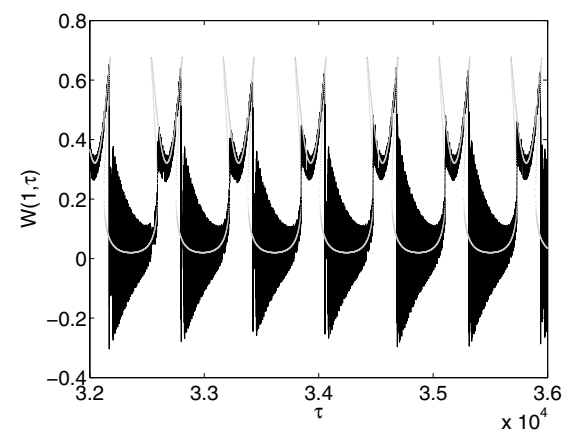

(b) $Y=0.5$

Fig. 6. Numerical solutions of Eq.(12), for $Z=10 \mathrm{~nm}$, in black and the approximated slow manifolds in grey

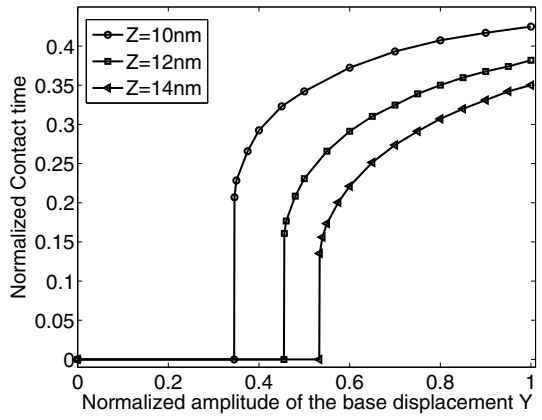

Fig. 7. Normalized contact time versus $Y$ for various initial gaps

time contact takes place through a saddle-node bifurcation that causes a jump from zero. This jump is decreasing by increasing the initial gap $Z$. Furthermore, the normalized contact time tends asymptotically to 0.5 for higher amplitudes $Y$, which means that the tip will spend $50 \%$ of the time in contact with the sample.

\section{Conclusion}

In this paper, we developed a mathematical model of an AFM microbeam subjected to a slow harmonic base motion and Lennard-Jones forces. We investigated the static contact and noncontact configurations and the associated natural frequencies and modes shapes. It was shown that the fundamental natural frequency near the contact mode is the most affected by the intermolecular interactions. Then, a one mode Galerkin method was employed and the equations of motion were transformed to a fast-slow dynamical system. It was found that the contact and the noncontact slow manifolds govern the dynamics of the AFM. Thus, we showed that the tapping mode is triggered via two saddlenode bifurcations of these slow manifolds. Moreover, the contact time between the tip and the sample was computed and the influences of the base motion amplitude and the initial gap discussed.

As a continuation of the present work the effects of higher modes is under investigation. The use of other types of interaction forces between the tip and the sample is planned.

\section{References}

1. Binnig G, Quate CF, Gerber CH. Atomic force microscope. Phys Rev Lett 56(9):930-934, 1986.

2. Jena BP, Heinrich Hörber JK. Force microscopy. Applications in biology and medicine. Wiley-Liss; 2006.

3. Eaton P, West P. Atomic force microscopy. Oxford university press; 2010.

4. Israelachvili J. Intermolecular and surface forces. 3rd edn. Academic Press; 2011.

5. Kirrou I, Belhaq M. Contact stiffness modulation in contact-mode atomic force microscopy. International Journal of Non-Linear Mechanics 55: 102-109, 2013.

6. Garcia R, Prez R. Dynamic atomic force microscopy methods. Surface science reports 47: 197-301, 2002.

7. Ashhab M, Salapaka MV, Dahleh M, Mezic I. Melnikov-based dynamical analysis of microcantilevers in scanning probe microscopy. Nonlinear Dyn 20: 197220, 1999.

8. Basso M, Giarrk L, Dahleh M, Mezic I. Complex dynamics in a harmonically excited Lennard-Jones oscillator: microcantilever-sample interaction in scanning probe microscopes. ASME J Dynam Syst Meas Control 122: 240-5, 2000.

9. Rützel S, Lee S, Raman A. Nonlinear dynamics of atomic-force-microscope probes driven in LennardJones potentials. Proc. R. Soc. Lond. A 459: 1925-1948, 2003.

10. Butt HJ, Cappella B, Kappl M. Force measurements with the atomic force microscope: technique, interpretation and applications. Surface Science Reports 59: 1-152, 2005.

11. Rosa-Zeiser A, Weilandt E, Hild S, Marti O. The simultaneous measurement of elastic, electrostatic and adhesive properties by scanning force microscopy: pulsedforce mode operation. Meas. Sci. Technol. 8: 1333-1338, 1997.

12. Pittenger B, Erina N, Su C. Quantitative mechanical property mapping at the nanoscale with PeakForce QNM. Bruker Application Note 128, 2010.

13. Verhulst F. Singular perturbation methods for slowfast dynamics. Nonlinear Dyn 50: 747-753, 2007.

14. Lakrad F, Schiehlen W. Effects of a low frequency parametric excitation. Chaos Solitons $\mathcal{F}$ Fractals 22: 1149-1164, 2004.

15. Lakrad F. Invariant slow manifolds of an Atomic Force Microscope system under the effects of Lennard-Jones forces and a slow harmonic base motion. Commun. Nonlinear Sci Numer Simulat 32, 49-62, 2016.

16. Tamayo J, García R. Deformation, Contact Time, and Phase Contrast in Tapping Mode Scanning Force Microscopy. Langmuir 12: 4430-4435, 1996. 